\title{
Correlação da Distribuição espacial do Banco de Sementes de Plantas Daninhas com a Fertilidade dos Solos ${ }^{1}$
}

\author{
Correlation of Weed Between Spacial Distribution and Soil Fertility
}

\author{
SHIRATSUCHI, L.S. ${ }^{2}$, FONTES, J.R.A. ${ }^{3}$ e RESENDE, A.V. ${ }^{4}$
}

\begin{abstract}
RESUMO - O objetivo deste trabalho foi identificar correlações entre o banco de sementes das plantas daninhas e os atributos da fertilidade dos solos. As amostragens de solo para determinação do banco de sementes foram feitas simultaneamente à amostragem para a análise da fertilidade do solo, utilizando uma grade amostral regular de 20 x $20 \mathrm{~m}$. Os mapas de infestação e de atributos de fertilidade dos solos foram gerados pela técnica de interpolação por "krigagem". Os valores de correlação entre o banco de sementes de BRAPL e a saturação de aluminio (m\%), a saturação de bases (V\%) e o $\mathrm{pH}$ foram de, respectivamente, 0,84, $-0,83$ e -0,74. Portanto, quanto maior o valor de $\mathrm{m} \%$ e menores os valores de $\mathrm{V} \%$ e $\mathrm{pH}$, maior a infestação do banco de sementes. Resultado semelhante foi observado para COMBE, porém os valores de correlação foram menores $(-0,35 \mathrm{com} \mathrm{V} \%$ e $0,28 \mathrm{com} \mathrm{m} \%)$. O banco de sementes de CYPRO apresentou a mesma tendência da BRAPL $(0,57$ com $\mathrm{m} \%,-0,63 \mathrm{com} \mathrm{V} \%,-0,7$ com $\mathrm{Ca}$ e -0,64 com o pH). Conclui-se que na área experimental houve alta correlação entre $\mathrm{pH}$, $\mathrm{Ca}, \mathrm{V} \%$ e $\mathrm{m} \%$ e o banco de sementes de plantas daninhas.
\end{abstract}

Palavras-chave: agricultura de precisão, macronutrientes, variabilidade espacial.

\begin{abstract}
The aim of this work was to identify the correlation between soil attributes and weed seed bank. The soil samplings for weed seed bank and soil fertility were collected simultaneously in the field using a $20 \times 20$ meter grid. The weed seed bank and soil fertility maps were generated by the kriging interpolation technique. The correlation values between the seed bank of BRAPL and aluminum saturation ( $m \%)$, base saturation (V\%) and $p H$ were, respectively, 0.84, -0.83 and -0.74. Thus, the higher the value of $m \%$ and the lower the values of $V \%$ and $p H$, the higher the weed seed bank was. Similar result was observed for COMBE at lower correlation values (-0.35 with V\% and of 0.28 with $\mathrm{m} \%)$. The CYPRO seed bank showed the same tendency as BRAPL $(0.57$ for $\mathrm{m} \%,-0.63$ for $\mathrm{V} \%,-0.7$ for $\mathrm{Ca}$ and -0.64 for the $\mathrm{pH}$ ). It was concluded that there was a high correlation among $\mathrm{pH}, \mathrm{Ca}, \mathrm{V} \%$ and $\mathrm{m} \%$ and the weed seed bank.
\end{abstract}

Key words: precision farming, macronutrients, spatial variability.

\section{INTRODUÇÃO}

A maioria dos trabalhos que enfocam o mapeamento das plantas daninhas diz respeito ao mapeamento da flora emergente durante o período crítico de prevenção da interferência. No entanto, existe uma grande dificuldade em mapear a flora emergente das plantas daninhas durante o ciclo da cultura, pois sua emergência depende de vários fatores biológicos, ambientais e de manejo. Entre os fatores que interferem na emergência de plantas daninhas, podem-se citar o sistema de produção, o histórico de fertilidade e de manejo de solos, os herbicidas utilizados, a cultura e o clima (Benoit et al., 1989).

Recebido para publicação em 27.9.2004 e na forma revisada em 5.9.2005.

Eng.-agrônomo, M.S., Pesquisador, Embrapa Cerrados, BR020, Km18, Caixa Postal 08223, 73301-970 Planaltina-DF, <shozo@cpac.embrapa.br>; ${ }^{3}$ Eng.-agrônomo, DSc, Técnico de Nível Superior, Embrapa Cerrados, <roberto@cpac.embrapa.br>; ${ }^{4}$ Eng.-agrônomo, D.S., Pesquisador, Embrapa Cerrados, <alvaro@cpac.embrapa.br>. 
Williams II et al. (1999) observaram que há variabilidade espacial da infestação da flora daninha emergente ao longo do tempo, de forma que de uma safra para outra havia mudança do local de ocorrência das reboleiras das plantas daninhas, sendo essa mudança em função do clima, do manejo de solo e do tipo de herbicida utilizado na cultura.

Um possivel fator que interfere no mapeamento da flora de plantas daninhas é a época de sua efetuação, visto que a espacialização da infestação está condicionada à época de emergência das plântulas (Forcella, 2004). Além da época de realização do mapeamento, o tempo disponivel para executá-lo em grandes áreas pode ser limitante, pois existem estádios ideais para o controle das plantas daninhas (Cardina et al., 1997), comprometendo a confecção do mapa para tratamento localizado, uma vez que, se o tempo despendido para o mapeamento da infestação for grande, poderá ser tarde para se tomar alguma decisão de controle.

Diferentemente da flora, o banco de sementes das plantas daninhas possui uma estabilidade espacial e temporal (Williams II et al., 1999; Shiratsuchi, 2001). Quando feito adequadamente, o mapeamento do banco de sementes pode ser utilizado para previsão dos locais de infestações em cultivos posteriores (Cardina et al., 1997).

As principais dificuldades no mapeamento do banco de sementes são as metodologias estudadas, a germinação de sementes, que está condicionada à dormência destas, e a dependência de ambas a fatores ambientais e fisiológicos não conhecidos em sua plenitude.

A adoção do método de mapeamento de solos em grades regulares (grid) por produtores que utilizam o mapeamento de solos para aplicação localizada de fertilizantes permitiria, em uma única operação, mapear também o banco de sementes das plantas daninhas, separandose parte do solo amostrado para essa determinação.

Os mapas de atributos do solo podem ser aproveitados para se otimizar o mapeamento das plantas daninhas, pois, segundo alguns autores (Haustler \& Nordmeyer, 1995; Qiu et al., 1998; Gaadi \& Ayers, 1999), há correlação entre atributos do solo e a infestação das plantas daninhas. Por meio de técnicas geoestatísticas, é possivel otimizar a amostragem de plantas daninhas utilizando resultados de análises de atributos de solo para a confecção de mapas de tratamento herbicida (Qiu et al., 1998).

Se há correlação entre atributos dos solos e a população das plantas daninhas (Haustler \& Nordmeyer, 1995; Qiu et al., 1998; Gaadi \& Ayers, 1999), o mesmo deve ocorrer quando se correlaciona o banco de sementes das plantas daninhas a atributos do solo, uma vez que alguns pesquisadores (Cardina et al., 1996; Shiratsuchi, 2001) observaram correlação entre a flora e o banco de sementes de algumas espécies.

Considerando que estudos relatando correlação entre mapas de banco de sementes e atributos de fertilidade dos solos são raros no Brasil, o objetivo deste trabalho foi identificar possiveis correlações entre o banco de sementes de plantas daninhas e atributos da fertilidade dos solos para facilitar o mapeamento dessas plantas, utilizando ferramentas da agricultura de precisão.

\section{MATERIAL E MÉTODOS}

O trabalho foi realizado em área experimental da Embrapa Cerrados (207868 L, 8274251 N, Zona 23L, UTM), em área cultivada com milho sob irrigação por pivô central.

A amostragem foi georreferenciada pelo Sistema de Posicionamento Global (GPS) numa grade amostral regular de $20 \times 20 \mathrm{~m}$, totalizando 73 pontos amostrais. Além da amostragem na grade regular, foi feita uma transecção de $150 \mathrm{~m}$, retirando-se 30 amostras distanciadas de 5 em $5 \mathrm{~m}$ para melhorar o ajuste dos modelos aos dados dos semivariogramas em pequenas distâncias.

Para cada ponto da grade amostral, foram coletadas três subamostras de solo de 0 a $20 \mathrm{~cm}$ de profundidade, sendo uma parte utilizada para análise química e outra para determinação do banco de sementes de plantas daninhas. Os atributos de solo avaliados foram as quantidades de $\mathrm{Ca}\left(\mathrm{meq} \mathrm{cm}^{-3}\right), \mathrm{o} \mathrm{pH}\left(\mathrm{H}_{2} \mathrm{O}\right)$, a saturação de bases (V\%) e a saturação de alumínio (m\%). 
O banco de sementes foi determinado pela observação da emergência de plântulas em vasos colocados em casa de vegetação, realizando-se 30 avaliações a cada sete dias, durante o período de 2.10.2002 a 17.12.2003, segundo metodologia descrita por Wilson et al. (1985). O banco de sementes foi estimado pela identificação e quantificação das plantas emergidas, por espécie. A quantificação foi expressa em densidade de sementes por área $\left(\mathrm{n}^{\circ} \mathrm{m}^{-2}\right)$, após dois fluxos de emergência de plântulas existentes no período de avaliação.

As plantas escolhidas para a confecção dos mapas de distribuição espacial e posterior correlação com os atributos de fertilidade dos solos foram as de maior importância relativa (Mueller-Dombois \& Ellenberg, 1974). Nessa área experimental, os maiores indices de importância foram das espécies Brachiaria plantaginea (BRAPL), Commelina benghalensis (COMBE) e Cyperus rotundus (CYPRO). Na apresentação de resultados, foram selecionados os mapas que obtiveram as maiores correlações.

A análise geoestatística consistiu da quantificação da semivariância e modelagem dos dados espaciais, com posterior interpolação dos dados por krigagem ordinária em blocos, resultando em mapas de superficie de resposta com valores estimados a cada $5 \mathrm{~m}$. Os valores interpolados estimados pelo modelo foram correlacionados, totalizando 1.191 valores de densidade de sementes e de atributos da fertilidade dos solos.

Os mapas de infestação e dos atributos de fertilidade dos solos foram gerados por interpolação por krigagem, utilizando os parâmetros dos modelos ajustados aos semivariogramas calculados e modelados pelo programa GS+. No caso da determinação do banco de sementes das plantas daninhas, os modelos foram ajustados aos dados brutos de contagem do número de plântulas emergidas por vaso, que, em seguida, foram apresentadas na forma de mapas, em número de sementes por metro quadrado. Para os atributos da fertilidade dos solos, os valores dos resultados das análises do solo foram utilizados para a modelagem dos semivariogramas.

Os mapas de atributos do solo e de banco de sementes das plantas daninhas foram correlacionados pela correlação linear de Pearson, utilizando o programa Statistica.

\section{RESULTADOS E DISCUSSÃO}

Durante o período de avaliação, ocorreram dois fluxos de emergência relevantes. Um ocorreu nos primeiros 35 dias após o início da irrigação, e o outro, após a aplicação de $\mathrm{KNO}_{3}^{-}$, seis meses após o primeiro fluxo.

Os resultados da densidade de sementes e da análise de solos foram submetidos a uma análise de estatística descritiva previamente à análise geoestatística. Essa análise descritiva prévia dos dados é imprescindivel para o conhecimento detalhado do fenômeno. Para isso, foram calculados a média, a mediana, o desvio-padrão, os valores mínimo e máximo, o coeficiente de variação, a curtose e a assimetria (Tabela 1).

Tabela 1 - Estatística descritiva da densidade do banco de sementes de plantas daninhas (sementes $\mathrm{m}^{-2}$ ) e de atributos da fertilidade dos solos

\begin{tabular}{|c|c|c|c|c|c|c|c|c|c|c|c|c|c|}
\hline \multirow{3}{*}{ Estatística } & \multicolumn{3}{|c|}{ Sementes $\mathrm{m}^{-2}$} & \multicolumn{10}{|c|}{ Atributo de fertilidade do solo } \\
\hline & \multirow{2}{*}{ BRAPL } & \multirow{2}{*}{ COMBE } & \multirow{2}{*}{ CYPRO } & $\mathrm{Ca}$ & $\mathrm{Mg}$ & $P$ & K & $\mathrm{Al}$ & SB & \multirow{2}{*}{$\frac{\mathrm{pH}}{\left(\mathrm{H}_{2} \mathrm{O}\right)}$} & V & $\mathrm{m}$ & $\begin{array}{c}\text { CTC } \\
\text { Efetiva }\end{array}$ \\
\hline & & & & \multicolumn{2}{|c|}{$\left(\right.$ meq cm $\left.{ }^{-3}\right)$} & \multicolumn{2}{|c|}{$\left(\mathrm{mg} \mathrm{dm}^{-3}\right)$} & \multicolumn{2}{|c|}{$\left(\mathrm{cmol}_{\mathrm{c}} \mathrm{dm}^{-3}\right)$} & & \multicolumn{2}{|c|}{$(\%)$} & $\left(\mathrm{cmol}_{\mathrm{c}} \mathrm{dm}^{-3}\right)$ \\
\hline Média & 133,09 & 64,97 & 14,64 & 2,94 & 0,77 & 88,90 & 185,00 & 0,71 & 4,11 & 5,03 & 32,66 & 16,03 & 12,71 \\
\hline Mediana & 21,90 & 11,30 & 3,36 & 3,01 & 0,78 & 89,40 & 180,00 & 0,64 & 4,00 & 5,05 & 33,91 & 13,80 & 12,80 \\
\hline Mínimo & 0,00 & 0,00 & 0,00 & 1,20 & 0,25 & 47,73 & 110,00 & 0,12 & 2,00 & 4,83 & 20,73 & 5,95 & 9,65 \\
\hline Máximo & $3.955,8$ & $1.804,40$ & 624,60 & 6,87 & 2,72 & 136,40 & 430,00 & 1,43 & 11,00 & 5,29 & 52,16 & 36,68 & 19,23 \\
\hline Desvio-padrão & 467,25 & 220,02 & 56,69 & 0,93 & 0,35 & 20,79 & 42,68 & 0,28 & 1,35 & 0,08 & 5,85 & 6,90 & 1,47 \\
\hline CV $(\%)$ & 351,09 & 338,63 & 387,19 & 31,54 & 45,43 & 23,37 & 22,96 & 39,29 & 32,96 & 1,67 & 17,92 & 43,04 & 11,55 \\
\hline Assimetria & 5,18 & 4,84 & 6,82 & 0,58 & 2,16 & 0,08 & 2,40 & 0,57 & 1,59 & $-0,31$ & $-0,06$ & 0,97 & 0,88 \\
\hline
\end{tabular}


Observaram-se amostras com elevado número de sementes, com um máximo de até 3.955 sementes $\mathrm{m}^{-2}$ (BRAPL) e outras sem sementes, resultando em valores de coeficientes de variação elevados (Tabela 1). Esse fato é indicativo do comportamento espacial de alto grau de contagiosidade (agregado) das plantas daninhas, resultando na ocorrência de manchas de infestações. Após a análise estatística, esse fenômeno foi confirmado pela confecção dos mapas da variabilidade espacial do banco de sementes das plantas daninhas.

O grande número de amostras com baixa densidade ou livre de sementes de plantas daninhas resultou em baixos valores de mediana. Cardina et al. (1996) observaram resultados semelhantes ao estudarem Chenopodim album.

Também foram observados valores elevados de coeficiente de variação para os parâmetros de solo, dando indicativos da ocorrência de manchas de fertilidade, o que foi confirmado pelos mapas gerados após análise geoestatística (Figuras 5 e 6).
Constatou-se que houve dependência espacial para a amostragem do banco de sementes das plantas daninhas (Tabela 2 e Figuras 1 e 2), a qual variou de 44 a 133 m, ou seja, amostragens realizadas a distâncias superiores a estas não são dependentes espacialmente entre si (Figuras 1 e 2). Essa informação auxilia a definir o tamanho da grade amostral para futura avaliação da área (Trangmar et al., 1985), para as espécies nela estudadas.

Para as três plantas daninhas estudadas, o modelo de melhor ajuste foi o esférico; portanto, ele foi adotado para o ajuste dos parâmetros dos semivariogramas. Para escolha do modelo foi realizada a validação cruzada com a infestação do banco de sementes das plantas daninhas (Tabela 2).

Os atributos dos solos possibilitaram o ajuste de parâmetros que demonstraram uma dependência espacial variando de 32 a $162 \mathrm{~m}$ (Tabela 3). Com esses valores de alcance, as amostragens realizadas a distâncias superiores a estas para cada atributo não são

Tabela 2 - Parâmetros dos ajustes dos semivariogramas adaptados aos modelos esféricos e da validação cruzada

\begin{tabular}{|c|c|c|c|c|c|c|}
\hline \multicolumn{5}{|c|}{ Semivariograma } & \multicolumn{3}{c|}{ Validação cruzada } \\
\hline Espécie & Intercepto & Patamar & Alcance $(\mathrm{m})$ & $\mathrm{r}^{2}$ & Coeficiente de regressão & $\mathrm{r}^{2}$ \\
\hline BRAPL & 0,1 & 297,0 & 133,60 & 0,93 & 0,93 & 0,65 \\
\hline COMBE & 24,2 & 48,5 & 111,20 & 0,86 & 0,11 & 0,42 \\
\hline CYPRO & 0,54 & 4,1 & 44,5 & 0,81 & 0,75 & 0,18 \\
\hline
\end{tabular}
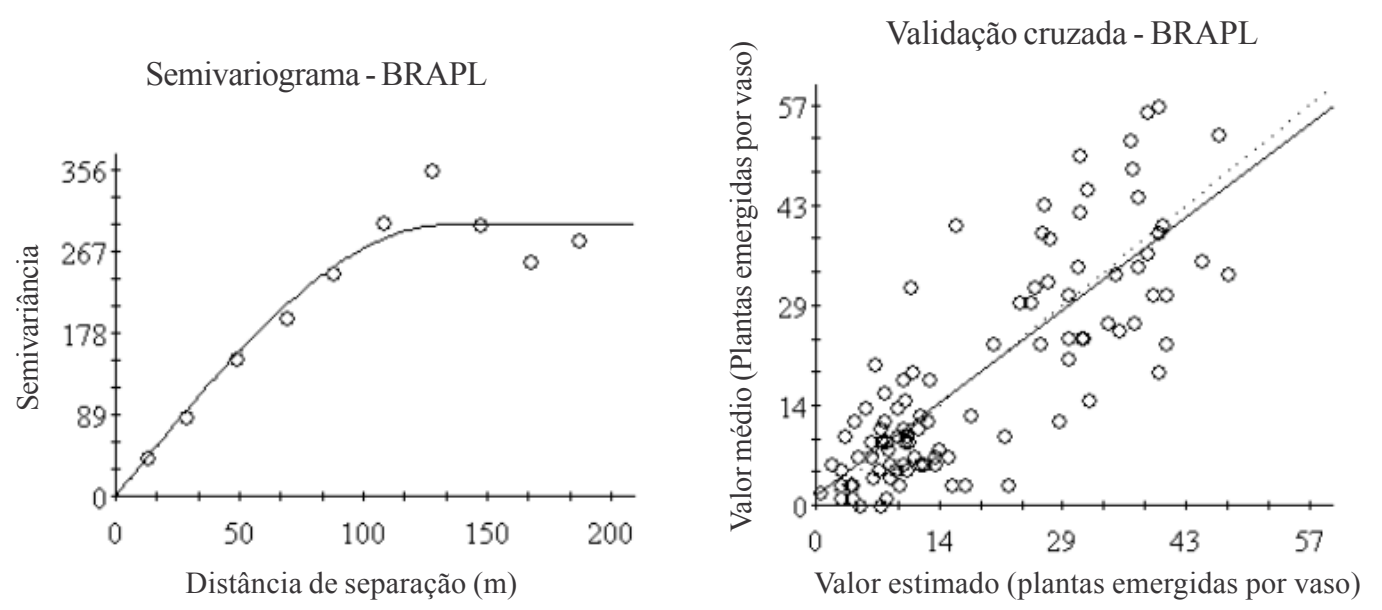

Figura 1 - Semivariograma ajustado do banco de sementes de BRAPL, seguido da validação cruzada. 

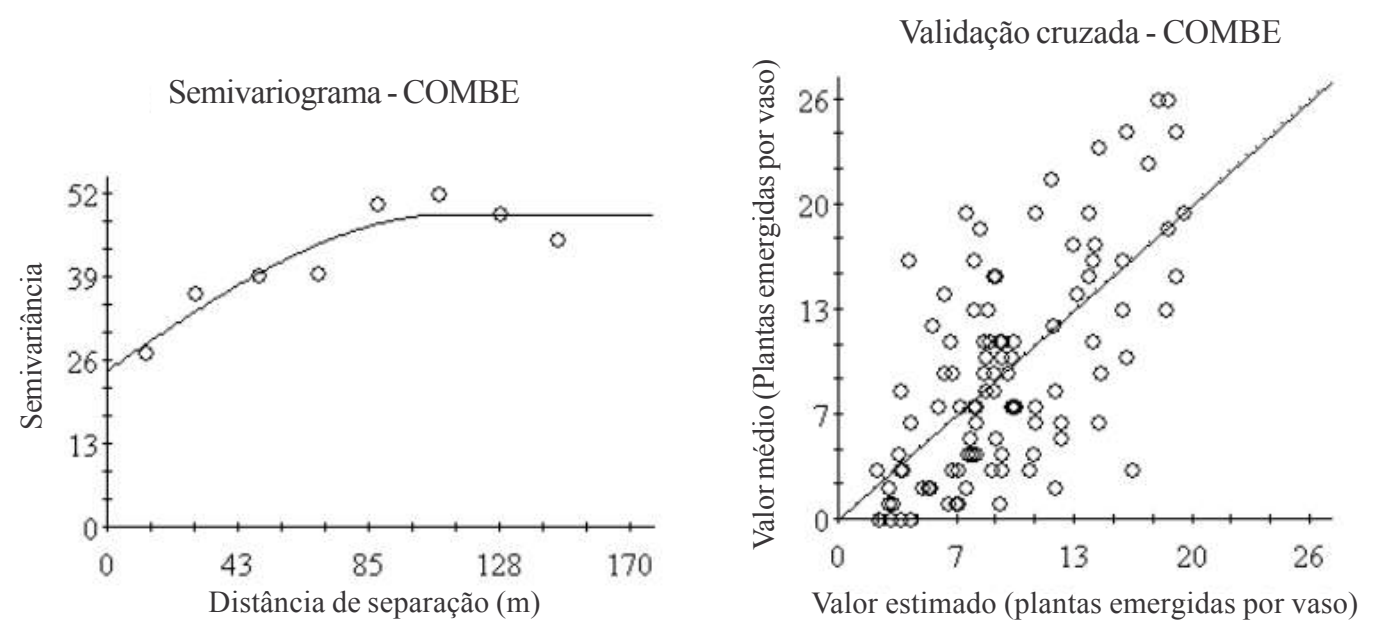

Figura 2 - Semivariograma ajustado do banco de sementes de COMBE, seguido da validação cruzada.

Tabela 3 - Parâmetros dos ajustes dos semivariogramas adaptados aos modelos esféricos e da validação cruzada

\begin{tabular}{|c|c|c|c|c|c|c|}
\hline \multicolumn{9}{|c|}{ Semivariograma } & \multicolumn{3}{c|}{ Validação cruzada } \\
\hline Atributo & Intercepto & Patamar & Alcance $(\mathrm{m})$ & $\mathrm{r}^{2}$ & Coeficiente de regressão & $\mathrm{r}^{2}$ \\
\hline $\mathrm{Al}$ & 0,01 & 0,08 & 83,0 & 0,78 & 1,03 & 0,51 \\
\hline $\mathrm{V} \%$ & 9,0 & 41,47 & 70,7 & 0,97 & 1,03 & 0,50 \\
\hline $\mathrm{pH}$ & 0,003 & 0,02 & 63,6 & 0,98 & 1,03 & 0,25 \\
\hline $\mathrm{P}$ & 108,0 & 482,0 & 163,8 & 0,96 & 0,98 & 0,44 \\
\hline $\mathrm{Ca}$ & 0,15 & 0,59 & 72,5 & 0,99 & 0,96 & 0,47 \\
\hline $\mathrm{Mg}$ & 0,015 & 0,045 & 32,0 & 0,74 & 1,11 & 0,50 \\
\hline $\mathrm{K}$ & 1,0 & $1.521,0$ & 33,7 & 0,92 & 0,50 & 0,10 \\
\hline
\end{tabular}

dependentes espacialmente entre si. Essa informação auxilia a adoção de grade amostral futura nesta área para o mapeamento dos atributos dos solos (Tabela 3). Além disso, a informação sobre a dependência espacial pode auxiliar a amostragem aleatória, para melhor representatividade do valor médio desses atributos na área agrícola. Esse tipo de informação pode auxiliar a amostragem tradicional de solos, pois, se o alcance da dependência espacial for de $162 \mathrm{~m}$, deve-se amostrar o solo nesta área em distâncias superiores a esta, a fim de que as amostras não sejam dependentes espacialmente.

Com os modelos esféricos também se obtiveram os melhores ajustes dos dados de fertilidade dos solos, sendo os coeficientes de regressão de 0,5 a 1,11 (Tabela 3 e Figuras 3 e 4).
Os valores do banco de sementes das três espécies de plantas daninhas e os atributos da fertilidade dos solos ( $\mathrm{pH}, \mathrm{Ca}, \mathrm{Mg}, \mathrm{K}, \mathrm{P}$, Soma de Bases, V\%, m\% e CTC) obtiveram correlações significativas a $1 \%$ de probabilidade, exceto BRAPL e teor de P no solo (Tabela 4); portanto, o banco de sementes de BRAPL foi independente do teor de $\mathrm{P}$ no solo nos niveis em que se encontrava a área de estudo.

Para as três espécies, houve correlação positiva entre banco de sementes, teor de $\mathrm{K}$ e saturação de alumínio (m\%) e correlação negativa com os demais atributos de fertilidade do solo (Tabela 4).

Observou-se comportamento diferenciado da infestação das plantas daninhas no que se refere ao teor de P. Enquanto a ocorrência do banco de sementes de BRAPL foi independente

Planta Daninha, Viçosa-MG, v. 23, n. 3, p. 429-436, 2005 
do teor de $\mathrm{P}$, a infestação de COMBE foi tanto maior quanto menor o teor deste elemento, e a de CYPRO, tanto maior quanto maior o teor de P (Tabela 4).

A espécie BRAPL apresentou correlações de 0,84 com m\% (Tabela 4 e Figura 5), -0,83 com $\mathrm{V} \%$ e -0,74 com o $\mathrm{pH}$; portanto, quanto

Semivariograma - Alumínio

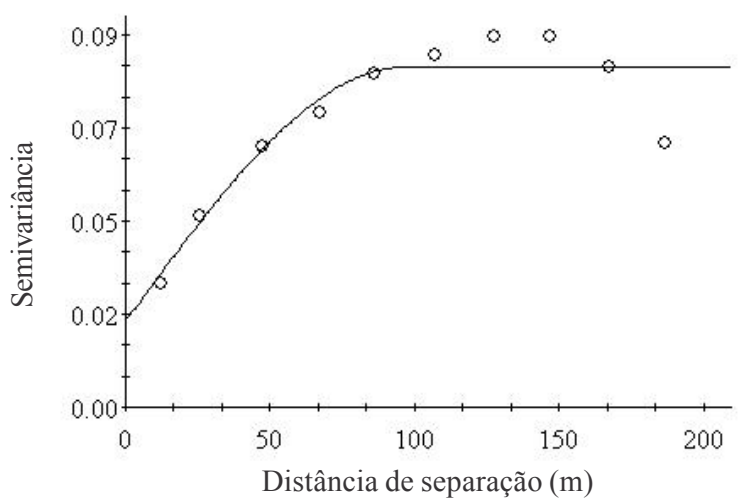

maior o $\mathrm{m} \%$ e menor a $\mathrm{V} \%$ e o $\mathrm{pH}$, maior foi a infestação do banco de sementes de BRAPL, o que explica o comportamento da espécie que se desenvolve nas mais variadas condições de solo. Os valores de correlação da espécie COMBE foram semelhantes aos da BRAPL, porém em menor intensidade (Tabela 4 e Figura 6) (-0,35 com a V\% e 0,28 com o m\%). O

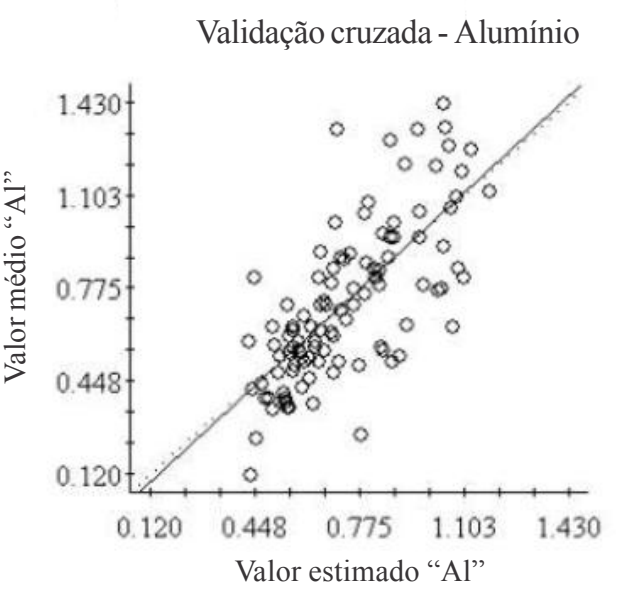

Figura 3 - Semivariograma ajustado a alumínio, seguido da validação cruzada.

Semivariograma - V\%

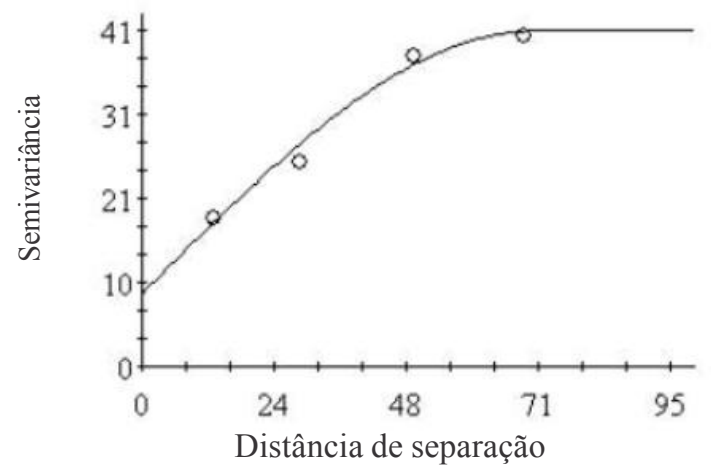

Validação cruzada - V\%

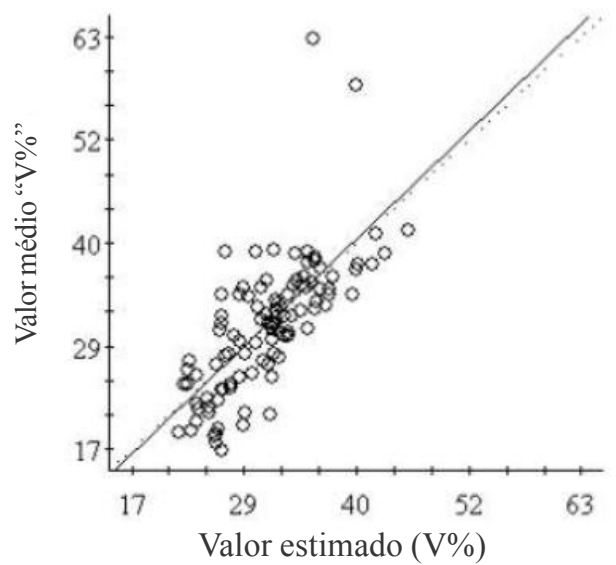

Figura 4 - Semivariograma ajustado à saturação de bases (V\%), seguido da validação cruzada.

Tabela 4 - Correlação linear entre o banco de sementes de plantas daninhas e os atributos da fertilidade do solo

\begin{tabular}{|l|c|c|c|c|c|c|c|c|c|}
\hline & $\mathrm{Ca}$ & $\mathrm{Mg}$ & $\mathrm{K}$ & $\mathrm{P}$ & $\mathrm{pH}$ & $\mathrm{SB}(\%)$ & $\mathrm{CTC}$ & $\mathrm{V} \%$ & $\mathrm{M} \%$ \\
\hline BRAPL & $-0,73^{*}$ & $-0,76^{*}$ & $0,55^{*}$ & $-0,01$ & $-0,74^{*}$ & $-0,75^{*}$ & $-0,61^{*}$ & $-0,83^{*}$ & $0,84^{*}$ \\
\hline COMBE & $-0,28^{*}$ & $-0,46^{*}$ & $0,61^{*}$ & $-0,12^{*}$ & $-0,09^{*}$ & $-0,33^{*}$ & $-0,17^{*}$ & $-0,35^{*}$ & $0,29^{*}$ \\
\hline CYPRO & $-0,70^{*}$ & $-0,56^{*}$ & $0,10^{*}$ & $0,36^{*}$ & $-0,64^{*}$ & $-0,68^{*}$ & $-0,76^{*}$ & $-0,63^{*}$ & $0,57^{*}$ \\
\hline
\end{tabular}

* Significativos a $1 \%$ de probabilidade. 

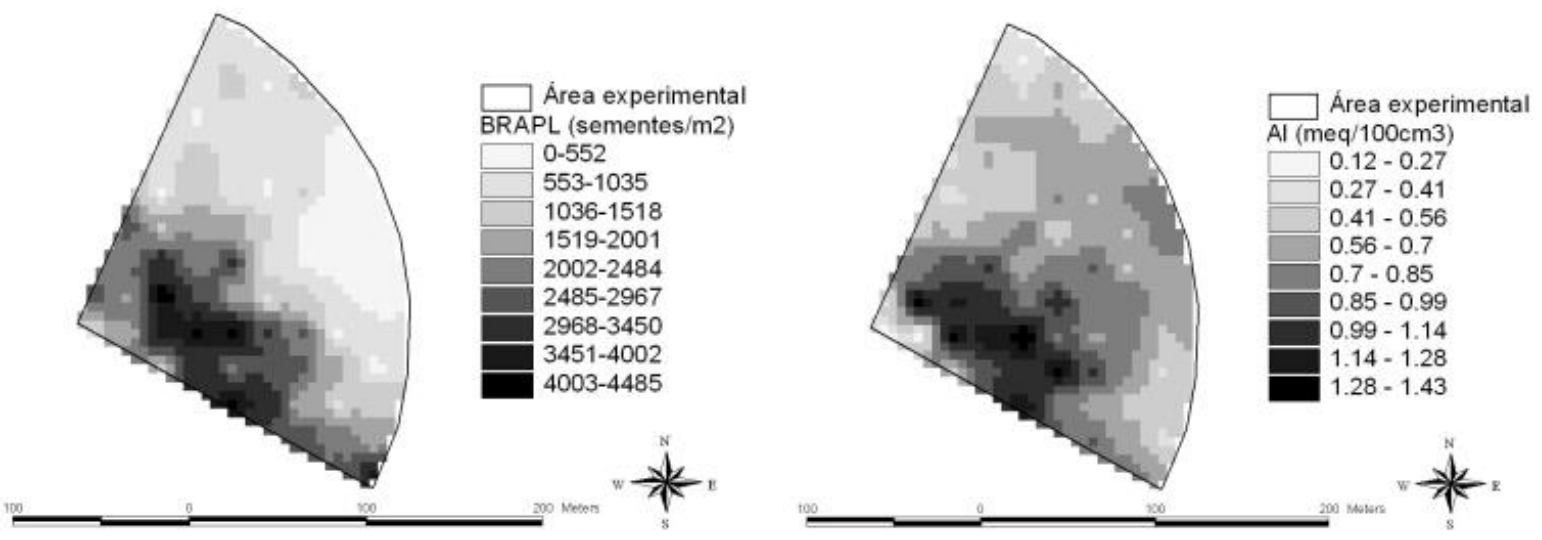

Figura 5 - Mapa interpolado da infestação do banco de sementes de BRAPL e da saturação de alumínio.
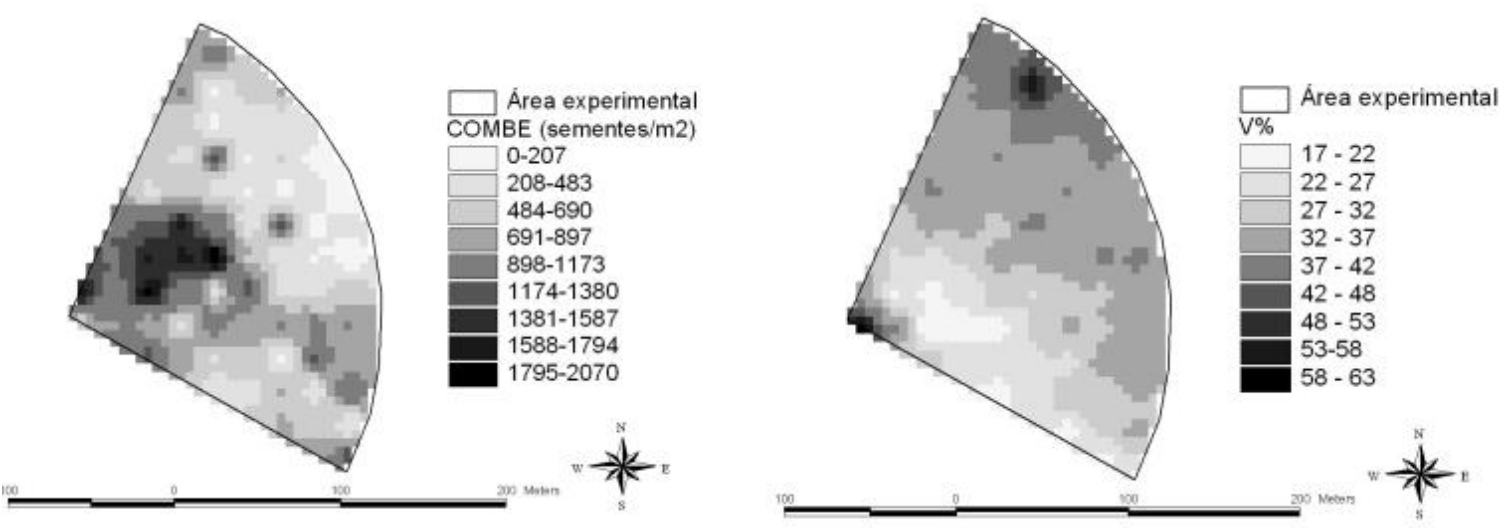

Figura 6 - Mapa interpolado da infestação do banco de sementes de COMBE e da saturação de bases.

banco de sementes de CYPRO também apresentou a mesma tendência daquele da BRAPL $(0,57$ com $\mathrm{m} \%,-0,63$ com a V\%, $-0,7$ com Ca e $-0,64$ com o $\mathrm{pH})$.

Dessa forma, nessa área experimental houve alta correlação entre os atributos de fertilidade dos solos e o banco de sementes de plantas daninhas, concluindo-se que podem ser utilizados os mapas de solos como subsídio para a geração de mapas do banco de sementes de BRAPL, COMBE e CYPRO. O inverso também é verdadeiro, isto é, pode-se utilizar o mapeamento do banco de sementes das plantas daninhas como subsídio na geração de mapas de alguns atributos dos solos.

\section{LITERATURA CITADA}

BENOIT, B. L.; KENKEL, N. C.; CAVERS, P. B. Factors influencing the precision of soil seed bank estimates. Can. J. Bot., v. 67, p. 2833-2840, 1989.
CARDINA, J.; JOHNSON, G. A.; SPARROW, D. H. The nature and consequence of weed spatial distribution. Weed Sci., v. 45, n. 3, p. 364-373, 1997.

CARDINA, J.; SPARROW, D. H.; Mc COY, E. L. Spatial relationships between seed bank and seedling populations of common lambsquarter (Chenopodium album) and annual grasses. Weed Sci., v. 44, p. 298-308, 1996.

FORCELLA, F. Site specific management guidelines: SSMG-20: Estimating the timing of weed emergence. Available: <http://www.ppi-far.org/ssmg>. Access: 22 mar. 2004.

GAADI, A.; AYERS, P. D. Integrating GIS and GPS into a spatially variable rate herbicide application system. Appl. Eng. Agric., v. 15, n. 4, p. 255-262, 1999.

HAUSTLER, A.; NORDMEYER, H. Inpact of soil on weed distribution. In: SEMINAR OF SITE SPECIFIC FARMING, Aarhus, 1995. Proceedings... Aarhus: Staten/ Planteavsforsog, 1995. p. 186-189.

Planta Daninha, Viçosa-MG, v. 23, n. 3, p. 429-436, 2005 
MUELlER-DOMBOIS, D.; ELLENBERG, H. A. Aims and methods of vegetation ecology. New York: John Wiley, 1974. $547 \mathrm{p}$.

QIU, W. et al. A feasibility study of a direct injection for variable rate herbicide application. Trans. Am. Soc. Agric. Eng., v. 40, n. 2, p. 291-299, 1998.

SHIRATSUCHI, L. S. Mapeamento da variabilidade espacial das plantas daninhas com a utilização de ferramentas da agricultura de precisão. $2001.96 \mathrm{f}$. Dissertação (Mestrado em Fitotecnia) - Escola Superior de Agricultura “Luiz de Queiroz” Piracicaba, 2001.
TRANGMAR, B. B.; YOST, R. S.; UEHARA, G.

Application of geostatistics to spatial studies of soil properties. Adv. Agron., v. 38, p. 45-94, 1985.

WILLIAMS II, M. M. et al. Spatio temporal outcomes of site-specific weed management in maize. In: THE EUROPEAN CONFERENCE OF PRECISION AGRICUlture, 2., Odense, 1999. Proceedings... Shefield: Shefield Academic Press, 1999. p. 897-906.

WILSON, R. G.; KERR, E. D.; NELSON, L. A. Potential for using weed seed content in the soil to predict future weed problems. Weed Sci., v. 33, p. 171-175, 1985. 